Supporting Information

for

\title{
Tailoring Membrane Nanostructure and Charge Density for High Electrokinetic Energy Conversion Efficiency
}

Sofie Haldrup ${ }^{\dagger}$,Jacopo Catalano ${ }^{\dagger}$, Mogens Hinge ${ }^{\dagger}$, Grethe V. Jensen ${ }^{\ddagger \S}$, Jan S. Pedersen ${ }^{\ddagger}$, and Anders Bentien ${ }^{\dagger *}$

†Department of Engineering, Aarhus University, Hangoevej 2, 8200 Aarhus N, Denmark

${ }^{*}$ Interdisciplinary Nanoscience Center (iNANO) and Department of Chemistry, Aarhus

University, Gustav Wieds Vej 14, 8000 Aarhus C, Denmark.

*Corresponding Author: bentien@eng.au.dk

${ }^{\S}$ Present Addresses: Niels Bohr Institute, University of Copenhagen, Universitetsparken 5, 2100 Copenhagen, Denmark. 


\section{Ion Exchange Capacity from ${ }^{1} \mathrm{H}-\mathrm{NMR}$}

The SPS contents of the cast membranes after the swelling procedures have been analysed by ${ }^{1} \mathrm{H}-\mathrm{NMR}$ spectroscopy and are shown in Figure S1. Here the peak at $7.4 \mathrm{ppm}$ (Figure S1a) was chosen for the SPS: it represents the protons closest to the sulfonic group in the benzene ring. For nitrocellulose the best resolved peak is at $5.85 \mathrm{ppm}$ (Figure S1b) and represents the methine proton on $\mathrm{c} 3$ in the nitrocellulose ring. ${ }^{1}$ In Figure $\mathrm{S} 1 \mathrm{c}$ the spectra of the CSPS4 and C-SPS5 membranes are shown as example and both peaks at 7.4 ppm 5.85 ppm can be clearly distinguished. The spectra were deconvoluted using ACD Lab 1D NMR Processor software and the resulting areas were used to calculate the ion exchange capacity (iec $c_{\mathrm{NMR}}$ ). Because of the complexity of the nitrocellulose spectrum a calibration curve was necessary and it was made from samples with known SPS/nitrocellulose mass balances and shown in the inset of Figure S1c.

In the calculation of the $i e c_{\mathrm{NMR}}$ it is assumed that 2 out of 3 hydroxyl groups have been nitrated and corresponds to a N-content of $11 \mathrm{wt} \%$. However, the broad definition of the collodion composition $(8-12.3 \mathrm{wt} \% \mathrm{~N})$ introduces an experimental uncertainty on $i e c_{\mathrm{NMR}} \cdot{ }^{2} \mathrm{~A}$ sensitivity analysis of the influence of the nitration level gives as an example $i e c_{\mathrm{NMR}}=$

$0.90_{-0.117}^{+0.089} \mathrm{meq}^{-1}$ for the C-SPS4 membrane. As a consequence it is estimated that the total experimental uncertainty on the absolute value of $i e c_{\mathrm{NMR}}$ is of the order of $\pm 10 \%$. 

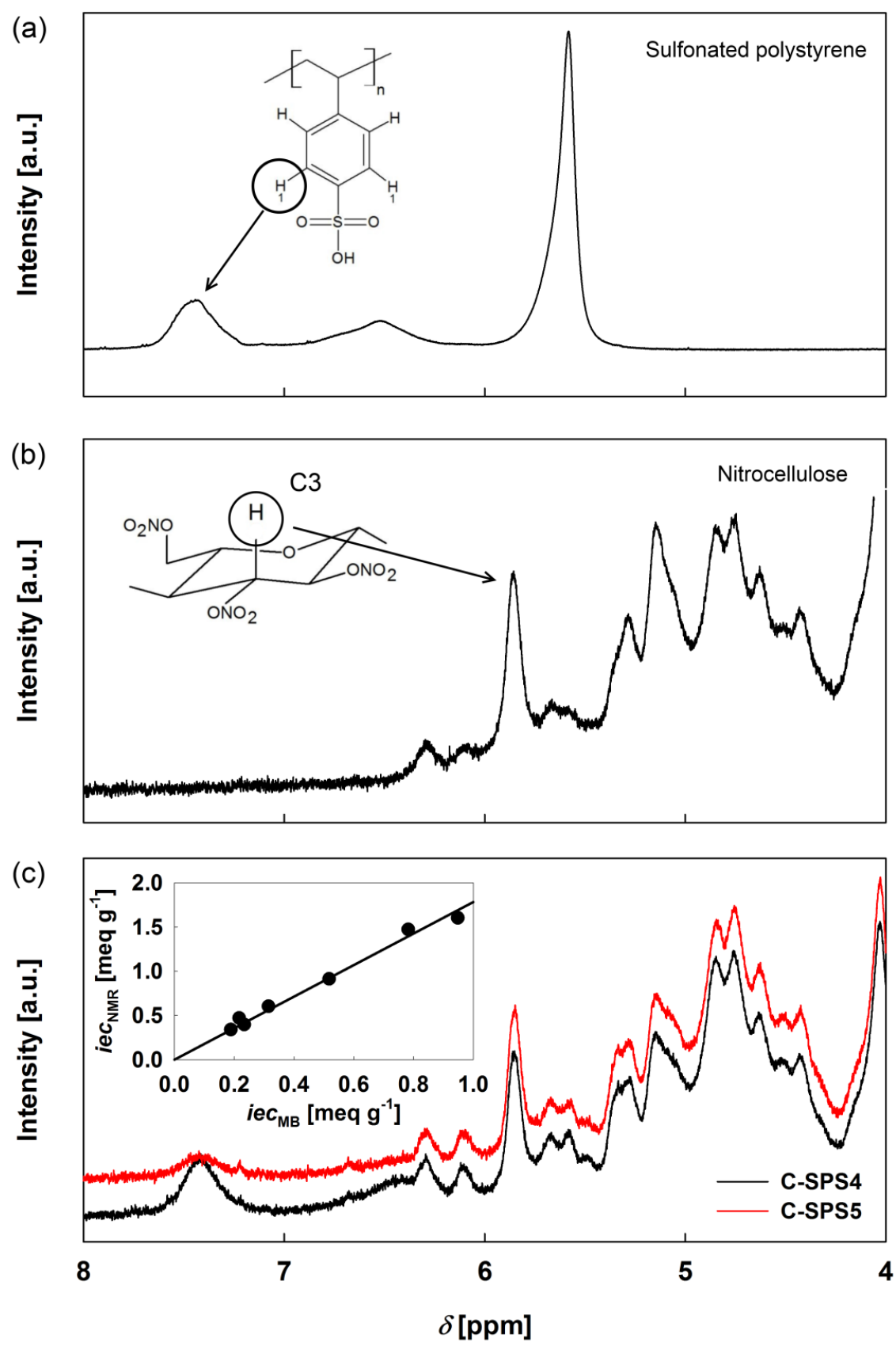

Figure S1. ${ }^{1} \mathrm{H}-\mathrm{NMR}$ spectra (Hz), from the top to the bottom: (a) sulfonated polystyrene, (b) nitrocellulose, and (c) C-SPS4 and C-SPS5 membranes. All samples have been dissolved in $\mathrm{Me}_{2} \mathrm{SO}_{4}-\mathrm{d}_{6}$ and the spectra acquired on a Bruker AVANCE III HD spectrometer running at 400 $\mathrm{MHz}$. The inset of (c) shows the calibration curve made of theoretical calculated iec $c_{\mathrm{MB}}$ from mass balances and the corresponding $i e c_{\mathrm{NMR}}$ determined from ${ }^{1} \mathrm{H}-\mathrm{NMR}$. 


\section{Small-Angle X-ray Scattering (SAXS)}

The structural model used to analyse the SAXS data is schematically depicted in Figure S2 and describes the pores as random-walk trajectories of overlapping poly-disperse spheres having an average diameter $d_{\mathrm{p}}$. The SAXS data and model fits are shown in Figure S3.

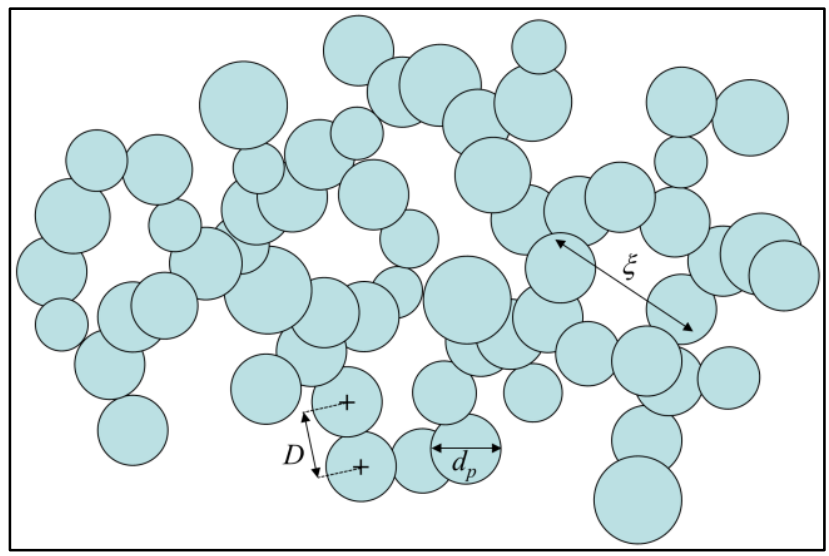

Figure S2. Structural model used for the SAXS data analysis describing the network of pores in the membrane.

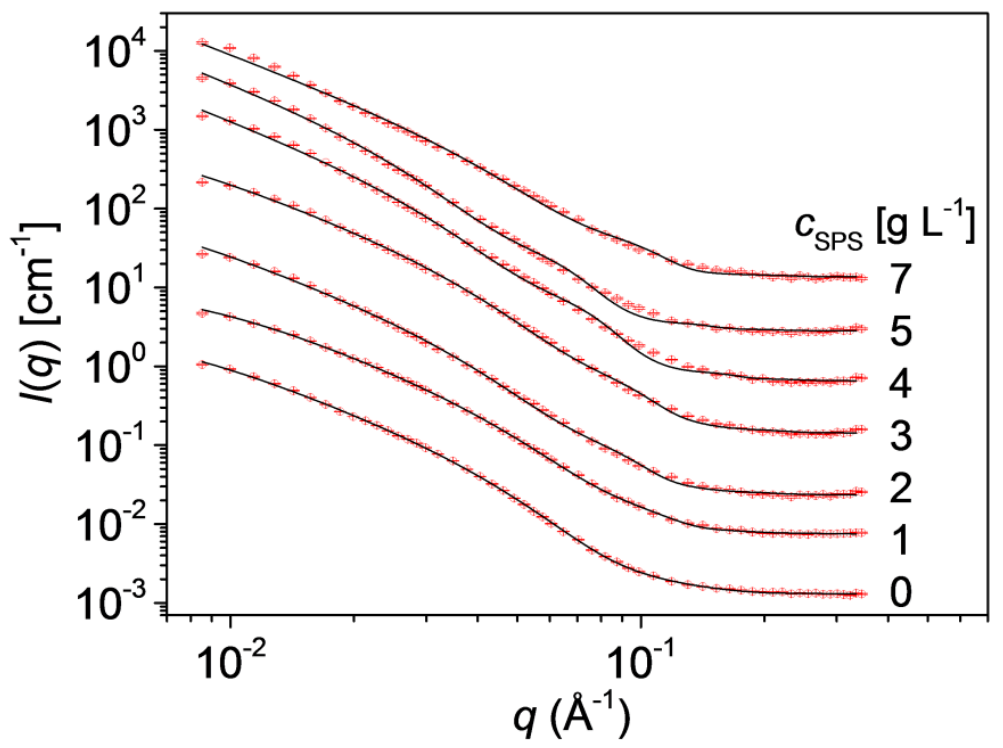

Figure S3. SAXS data (red points) with model fits (black lines) for the different concentrations of SPS, $C_{\mathrm{SPS}}$. The data for $C_{\mathrm{SPS}}=0$ are on absolute scale. For clarity, the other data sets are scaled by a factor of five for each consecutive value of $C_{\mathrm{SPS}}$. 
The model has been optimized for best possible fit to the experimental intensity data. The model intensity, $\boldsymbol{I}(\boldsymbol{q})$, is given by:

$I(q)=a\langle P(q)\rangle S(q)+b$

In which $a$ and $b$ are a scale factor and a constant background, respectively. $P(q)$ is the form factor giving the scattering from each sub-particle of the model and, for monodisperse spherical particles of radius $R$, it is given by ${ }^{2}$ :

$P(q)=[\Phi(q R)]^{2}$

where

$\Phi(q R)=\frac{3[\sin (q R)-q R \cos (q R)]}{(q R)^{3}}$

The sphere radius diameter is Schulz distributed with an average radius $\bar{R}$ and a relative width of the distribution $\sigma$. The Schulz distribution is given by:

$f(R)=\left[\frac{z+1}{\bar{R}}\right]^{z+1} R^{z} \exp \left[-\left(\frac{z+1}{\bar{R}} R\right)\right] \frac{1}{\Gamma(z+1)}$

where $z=1 / \sigma^{2}-1$ and $\Gamma(x)$ is the gamma function. The form factor was averaged over the size distribution (in the interval $\bar{R} \pm 10 \sigma \bar{R}$ ):

$$
\langle P(q)\rangle=\int_{R(1-10 \sigma)}^{R(1+10 \sigma)} f(R)[\Phi(q R)]^{2} d R
$$

The structure factor $S(q)$ introduces the effect of the relative positions of the subparticles. The spheres have centers on points of a random walk trajectory, and a structure factor is applied as described in Ref 3. The fit parameters for all the membranes are reported in Table S1. In 
particular the number of spheres in a random-walk aggregate $N$ is related to the mesh size of the sponge-like material: beyond a certain length scale above this size, the structure will appear homogeneous and hence cannot be described by a random walk of spheres. This length scale can be quantified as the correlation length for the sponge-like material, given by:

$$
\xi=\left(R_{\mathrm{g}}{ }^{2} / 3\right)^{0.5}
$$

where $R_{\mathrm{g}}$ is the radius of gyration of the random-walk chain, given by $R_{\mathrm{g}}=D(N / 6)^{0.5}$ and $D$ is the distance between the spheres in the random-walk.

Table S1 Parameters entering in the model used for analysing the SAXS data.

\begin{tabular}{cccccc}
\hline C-SPS & $d_{\mathrm{p}}$ & $\sigma$ & $\begin{array}{c}D \\
n m\end{array}$ & $N$ & $\xi$ \\
& $n m$ & & & $n m$ \\
\hline 0 & $5.1 \pm 0.4$ & $0.27 \pm 0.03$ & $5.0 \pm 0.4$ & $128 \pm 19$ & $13.4 \pm 1.6$ \\
1 & $5.7 \pm 0.1$ & $0.18 \pm 0.01$ & $5.4 \pm 0.2$ & $73 \pm 7$ & $10.8 \pm 0.6$ \\
2 & $6.7 \pm 0.2$ & $0.19 \pm 0.02$ & $6.7 \pm 0.3$ & $119 \pm 46$ & $17.2 \pm 3.4$ \\
3 & $6.4 \pm 0.2$ & $0.20 \pm 0.02$ & $6.5 \pm 0.3$ & $108 \pm 35$ & $15.8 \pm 2.7$ \\
4 & $7.9 \pm 0.2$ & $0.23 \pm 0.02$ & $8.6 \pm 0.4$ & $1000^{b}$ & $64.3^{c}$ \\
5 & $9.1 \pm 0.3$ & $0.24 \pm 0.02$ & $9.9 \pm 0.4$ & $1000^{b}$ & $73.6^{c}$ \\
7 & $5.9 \pm 0.3$ & $0.19 \pm 0.02$ & $5.7 \pm 0.2$ & $1000^{b}$ & $46.0^{c}$ \\
\hline
\end{tabular}

Notes: ${ }^{\text {a }}$ Parameter derived from $D$ and $N .{ }^{b}$ This parameter was fixed in the fit, as it falls beyond the resolution of the experiment. ${ }^{c}$ derived from the fixed parameter $N$ which falls beyond the resolution of the experiment of $\sim 50 \mathrm{~nm}$. 
Table S2 Main results for the membranes studied in the present work measured in $0.03 \mathrm{M} \mathrm{LiCl}$ solutions and at ambient temperature.

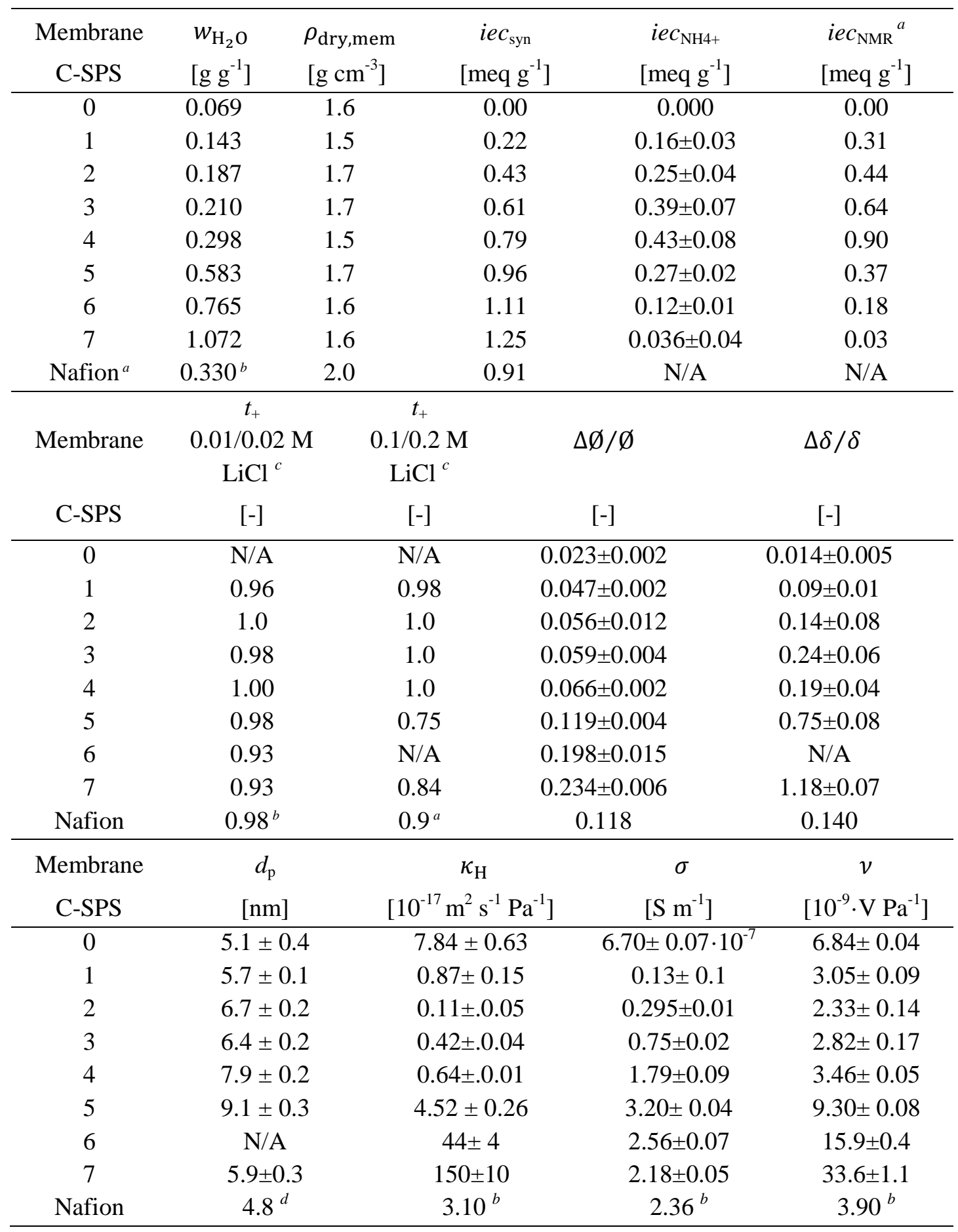

Notes: $a$ for estimates of the errors read the main text, $b$ Nafion 117 from Ref.4, $c$ the error in between experiments is below $2 \%$ for all the membranes, $d$ Nafion 120 from Ref. 5 for which the Bragg spacing in fully hydrated conditions has been considered. 
Table S3 Calculated volume swelling, intrinsic hydraulic permeability, charge density, electrokinetic figure-of-merit, efficiency and hydraulic and electrical power density. Power density has been calculated considering $\Delta p=1 \mathrm{MPa}$ and membrane thickness of $50 \mu \mathrm{m}$ for all the membranes reported.

\begin{tabular}{|c|c|c|c|c|c|c|c|}
\hline $\begin{array}{c}\text { Membrane } \\
\text { C-SPS }\end{array}$ & $\begin{array}{l}\frac{\Delta V}{V} \\
{[-]}\end{array}$ & $\begin{array}{c}\kappa_{\mathrm{H}}^{*} \\
{\left[\mathrm{~m}^{2} \mathrm{~s}^{-1} \mathrm{~Pa}^{-1}\right]}\end{array}$ & $\begin{array}{c}\Sigma \\
{\left[\mathrm{C} \mathrm{m}^{-2}\right]}\end{array}$ & $\begin{array}{c}\beta \\
{[-]} \\
\end{array}$ & $\begin{array}{c}\eta_{\max } \\
{[-]}\end{array}$ & $\begin{array}{c}\left(\frac{P}{A}\right)_{\mathrm{hy}} \\
{\left[\mathrm{W} \mathrm{m}^{-2}\right]}\end{array}$ & $\begin{array}{c}\left(\frac{P}{A}\right)_{\mathrm{el}} \\
{\left[\mathrm{W} \mathrm{m}^{-2}\right]}\end{array}$ \\
\hline 0 & 0.06 & $7.84 \cdot 10^{-17}$ & N/A & $4.0 \cdot 10^{-7}$ & $1.0 \cdot 10^{-7}$ & 1.56 & $1.6 \cdot 10^{-7}$ \\
\hline 1 & 0.19 & $1.00 \cdot 10^{-17}$ & 0.266 & $\underset{1}{1.38 \cdot 10^{-}}$ & 0.032 & 0.27 & $8.6 \cdot 10^{-3}$ \\
\hline 2 & 0.27 & $2.70 \cdot 10^{-18}$ & 0.459 & 1.47 & 0.223 & 0.04 & $8.4 \cdot 10^{-3}$ \\
\hline 3 & 0.402 & $1.01 \cdot 10^{-17}$ & 0.614 & 1.43 & 0.218 & 0.14 & 0.03 \\
\hline 4 & 0.36 & $2.78 \cdot 10^{-17}$ & 0.641 & 3.32 & 0.350 & 0.34 & 0.12 \\
\hline 5 & 1.19 & $3.22 \cdot 10^{-16}$ & 0.242 & 6.14 & 0.455 & 3.67 & 1.67 \\
\hline 6 & N/A & $1.09 \cdot 10^{-15}$ & N/A & 1.48 & 0.223 & 15.30 & 3.40 \\
\hline 7 & 2.32 & $3.94 \cdot 10^{-15}$ & 0.014 & 1.66 & 0.240 & 54.20 & 13.00 \\
\hline Nafion & 0.66 & $6.69 \cdot 10^{-17 a}$ & N/A & $1.16^{a}$ & $0.180^{a}$ & $0.9^{a}$ & $0.19^{a}$ \\
\hline
\end{tabular}

Notes: $a$ from Ref. 4.

\section{References}

1. Wu, T. K. Carbon-13 and Proton Nuclear Magnetic Resonance Studies of Cellulose Nitrates. Macromolecules 1980, 13, 74-79.

2. $\quad$ Rayleigh, L. The Incidence of Light Upon a Transparent Sphere of Dimensions Comparable with the Wave-Length. Proc. R. Soc. London, Ser. A 1910, 84, 25- 46.

3. Giehm, L.; Oliveira, C. L. P.; Christiansen, G.; Pedersen, J. S.; Otzen, D. E. Sds-Induced Fibrillation of A-Synuclein: An Alternative Fibrillation Pathway. J. Mol. Biol. 2010, 401, 115133.

4. Kilsgaard, B. S.; Haldrup, S.; Catalano, J.; Bentien, A. High Figure of Merit for Electrokinetic Energy Conversion in Nafion Membranes. J. Power Sources 2014, 247, 235242.

5. Gierke, T. D.; Munn, G. E.; Wilson, F. C. The Morphology in Nafion Perfluorinated Membrane Products, as Determined by Wide- and Small-Angle X-Ray Studies. J. Polym. Sci., Part B: Polym. Phys. 1981, 19, 1687-1704. 